\title{
Statistical experimental design screening strategies for free-monomeric isocyanates determination by UPLC in materials used in cork stoppers manufacturing
}

\author{
Catarina André ${ }^{1}$, Inês Delgado ${ }^{2}$, Isabel Castanheira ${ }^{2}$, João Bordado ${ }^{1}$, Ana Sofia Matos ${ }^{3}$ \\ ${ }^{1}$ Departamento de Engenharia Química, Instituto Superior Técnico, Universidade de Lisboa Avenida Rovisco Pais 1049-001 Lisboa, Portugal \\ ${ }^{2}$ Departamento de Alimentação e Nutrição, Instituto Nacional de Saúde Doutor Ricardo Jorge (INSA), Av. Padre Cruz 1649-016 Lisboa, \\ Portugal \\ ${ }^{3}$ UNIDEMI, Departamento de Engenharia Mecânica e Industrial, Faculdade de Ciências e Tecnologia, Universidade Nova de Lisboa, $2829-516$ \\ Caparica, Portugal
}

\begin{abstract}
A statistical experimental design was used to screen variables of the analytical procedure to quantify free monomeric isocyanates presented in polyurethane based pre-polymers in trace amounts.

For this purpose, diphenylmethane-4,4'-diisocyanate (4,4'-MDI), 2,4-toluene diisocyanate (2,4-TDI) and 2,6-toluene diisocyanate (2,6-TDI) were analysed by Ultra Performance Liquid Chromatography with a Photo Diode Array detector (UPLC-PDA). A preliminary study was performed with three derivatization agents, being 1-(2-piridyl) piperazine (1,2-PP) the most suitable one. Column temperature, flow and percentage of ammonium acetate (\% NH4Ac.) were the factors studied at two levels each. A sequence of experiments was planned according to a $2^{3}$ full factorial design with three replicates and two repetitions. Analysis of variance (ANOVA) was applied for the identification of significant factors and interactions.

Higher responses were achieved when the column temperature was $30^{\circ} \mathrm{C}$, a flow of $0.3 \mathrm{~mL} \mathrm{~min}{ }^{-1}$ and a solvent with a percentage of ammonium acetate of $0.1 \%$. Figures of merit were assessed within-laboratory as a preliminary step for method validation. Similar values were obtained for TDI and MDI. Recoveries are approximately $100 \%$. In addition, the values of detection limits (LODs) for MDI and TDI were 0.08 and $0.11 \mu \mathrm{g} \mathrm{m}^{-1}$, respectively, and quantification limits (LOQs) were 0.25 and $0.33 \mu \mathrm{g} \mathrm{mL}$ for MDI and TDI, respectively. The working range was between 0.01 and $10.00 \mu \mathrm{g} \mathrm{mL}^{-1}$ for $\mathrm{MDI}$ and $0.01-4.95 \mu \mathrm{g} \mathrm{mL}^{-1}$ for TDI. These figures of merit seemed adequate to detect low amounts of free monomeric isocyanates presented in agglomerates and foams for agglomerated cork stoppers production. This data is suitable to address the optimization of an analytical method by a response surface methodology.
\end{abstract}

\section{Section: RESEARCH PAPER}

Keywords: diisocyanates; derivatization; chromatography; design of experiments; cork stoppers

Citation: Caratina André, Inês Delgado, Isabel Castanheira, João Bordado, Ana Sofia Matos, Statistical experimental design screening strategies for freemonomeric isocyanates determination by UPLC in materials used in cork stoppers manufacturing, Acta IMEKO, vol. 6, no. 1, article 8, April 2017, identifier: IMEKO-ACTA-06 (2017)-01-08

Section Editor: Janaína Marques Rodrigues, INMETRO, Brazil

Received June 15, 2016; In final form November 18, 2016; Published April 2017

Copyright: (C) 2017 IMEKO. This is an open-access article distributed under the terms of the Creative Commons Attribution 3.0 License, which permits unrestricted use, distribution, and reproduction in any medium, provided the original author and source are credited

Funding: This work was supported by project QREN 5012-LIRACork

Corresponding author: Ana Sofia Matos, e-mail: asvm@fct.unl.pt

\section{INTRODUCTION}

Cork stoppers are considered the premium material for sealing bottled wine. The main reasons for this are their high compressibility, flexibility and impermeability, which prevent wine from oxidation and deterioration. Moreover, cork stoppers are a natural product and therefore environmentally friendly. To avoid wasting residues from the manufacturing process, some sub-products, such as agglomerated cork stoppers, can be produced. The granulated cork is used together with binders that, according to the International Code 
of Cork Stoppers Manufacturing Practices [1], must be polyurethane based (food grade). During manufacture, residual unpolymerized isocyanate monomer can remain in the polymer and may migrate into wine [2].

Isocyanates are very reactive chemical compounds, which contain one or more functional groups of the type $-\mathrm{N}=\mathrm{C}=\mathrm{O}$. These compounds are classified regarding the number of functional groups contained. They may be mono-, di- or polyisocyanates according to containing one, two or more groups, respectively (Figure 1 a) b) c) d) e)).

There is a critical effect on health associated when these compounds are found in the working environment at high doses that may cause irritation of eyes, skin and respiratory system. For this reason, workers in this type of industry should take special care to protect the eyes, skin and inhalation of vapours. A high care for humans, after long periods of exposure, may become sensitive to extremely low concentrations. Coughing, wheezing, chest discomfort, edema and interstitial pulmonary fibrosis are some of the effects that have occurred associated with inhalation of isocyanates. The effect that these compounds have on health are, therefore, very well described in the specific documentation on this subject where the limits for exposure to these compounds are also documented [3]. Due to the health effects, these limits are low in some European countries $\left(<20 \mathrm{mg} \mathrm{L}^{-1}\right)$.

High performance liquid chromatography has been widely employed for the analysis of isocyanates [2], [4]-[6]. However, this technique requires long run times and large amounts of solvents.

The emerging of ultra-performance liquid chromatography (UPLC) was an important advance in chromatography performance. The improvement of this new technique was the use of columns packed with particles smaller than $2 \mu \mathrm{m}$. The van Deemter equation indicates that as the particle size decreases to less than $2.5 \mu \mathrm{m}$, there is a significant gain in efficiency and that efficiency does not diminish at increased flow rates or linear velocities [7], [8]. On the other hand, with the decrease of particle size the operating pressure increases and a decreasing of flow rates can be used. With this progress, it is possible to decrease the theoretical plate height that allows overall separation with better resolution. Due to the high reactivity of isocyanates with water derivatization of isocyanates is crucial. In literature several derivatization agents were reported. The derivatization reagents most frequently used are

a)
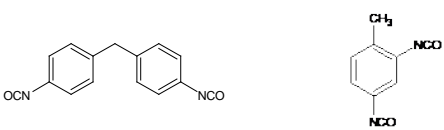

d)

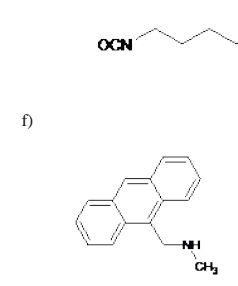

Figure 1. Structure of isocyanates and derivatization agents. a) 4,4'diphenylmethane diisocyanate; b) 2,4-toluene diisocyanate; c) 2,6-toluene diisocyanate; d) Hexamethylene diisocyanate; e) 1-Naphthyl isocyanate; f) 9-(N-methylaminomethyl) anthracene; g) 1-(2-pyridyl) piperazine; h) dibutylamine.
MAMA, 1,2-PP, DBA among others (Figure $1 \mathrm{f}$ g) h)) [9].

The ability of a chromatographic method to separate, identify and quantify compounds satisfactorily depends on several factors which can be controlled by the operator. Often the use of the design of experiments (DOE) can be a powerful tool, which allows the analyst to identify which factors or interaction of factors can affect the chromatographic method, driving to optimization [10]-[14]. Whatever the application area, the design of experiments ensure that results are obtained in a more effective way, since it considers the variation of the factors in combination, and their interactions [15]-[17].

The design of experiments allows structuring the order of trials to translate the objectives predefined by the investigator. The DOE has two applications in chromatography. The first is to show that no factors in the study are significant, and thus verify the robustness of the method for validation. The second one, used in this study, is to identify which factors are significant and optimize the response taking into account these same factors [12]. Analysis of variance (ANOVA) is used for the statistical treatment of results after performing the experiments. This variance analysis allows identifying, objectively, which factors and/or interactions are significantly affecting the answers. After identifying these factors (alone or in interaction), it establishes the best combination of factor levels that will lead to the maximization of the pre-established objectives.

As far as we known UPLC methods are not used to quantify isocyanates at low concentration levels $\left(<1 \mathrm{mg} \mathrm{kg}^{-1}\right)$. In our previous work [18], the Taguchi method was applied for the determination of free isocyanate analysis in UPLC, using MAMA as a derivatization agent. However, MAMA seems not suitable for low concentrations due to a higher limit of detection [19].

The main goal of this study was to evaluate the effect of three controllable factors in a chromatographic method to determine free diisocyanates at low concentration levels present in cork stoppers, by a screening process.

\section{MATERIALS AND METHODS}

\subsection{Reagents and Chemicals Standards}

All solvents used were HPLC or superior grade with purity higher than $96 \%$. 2,4-toluene diisocyanate (2,4-TDI) (96\%), 2,6-toluene diisocyanate (2,6-TDI) (98.5\%), hexamethylene diisocyanate (HDI) $(99 \%)$ from Ehrenstorfer; mixture of 2,4toluene diisocyanate $(80 \%)$ and 2,6-toluene diisocyanate $(20 \%)$ (T80) from Bayer; diphenylmethane-4,4'-diisocyanate (4,4'MDI) (98\%), 9-(methylaminomethyl)anthracene (MAMA) (99 $\%)$ 1-(2-piridyl)piperazine (1,2-PP) (99.5\%), dibutylamine (DBA) $(99.5 \%)$ from Aldrich; triethylamine (TEA) (99\%) from Alfa Aesar; Dimethyl sulfoxide (DMSO), from SigmaAldrich; dichloromethane (DCM), acetonitrile (AcN), ammonium acetate (NH4Ac.), formic acid (98-100\%), isooctane, ethanol from Merck; orthophosphoric acid (85\%), glacial acetic acid from Panreac; N-N'-dimethylformamide (DMF) from Emsure. The ultra-pure water used for all purposes was obtained with a Milli-Q Element system from Millipore (Interface, Portugal).

\subsection{Derivatization and sample preparation procedures}

\subsubsection{MAMA - derivatives}

MAMA derivatives were prepared at a concentration of 100 $\mu \mathrm{g} \mathrm{mL} \mathrm{mL}^{-1}$ in DCM. Working standards were derivatized with 
MAMA for one hour in the dark, evaporated to dryness in nitrogen flow and redissolved in $10 \mathrm{~mL}$ of DMF: mobile phase 50:50.

\subsubsection{1,2-PP-derivatives}

1,2-PP derivatives were prepared at a concentration of 500 $\mu \mathrm{g} \mathrm{mL} \mathrm{m}^{-1}$ in DCM and derivatized with $50 \mu \mathrm{L}$ of 1,2-PP. These stock solutions were derivatized for one hour. Intermediate standard solutions were prepared, by dilution, at a concentration of $80 \mu \mathrm{g} \mathrm{mL}-1$ in AcN:DMSO (95:5). Working standards were prepared, by dilution with $\mathrm{AcN}$, to a concentration of $8 \mu \mathrm{g} \mathrm{mL} \mathrm{m}^{-1}$.

\subsection{3. $D B A$ - derivatives}

A solution of DBA was prepared in $\mathrm{AcN}$ at a concentration of $5000 \mu \mathrm{g} \mathrm{mL} \mathrm{m}^{-1}$. Isocyanate solution was prepared in isooctane at a concentration of $500 \mu \mathrm{g} \mathrm{mL}^{-1}$ and added dropwise to the DBA solution with continuous stirring. A rotating evaporator was used to evaporate this mixture to dryness. After removing DBA surplus under vacuum, the precipitate was recrystallized with $70 \%$ aqueous ethanol. Accurately weighed amounts of working solution were dissolved in acetonitrile at a concentration of $1000 \mu \mathrm{g} \mathrm{mL}-1$ and further diluted in $\mathrm{AcN}$ : water (50:50) at a concentration of $100 \mu \mathrm{g} \mathrm{mL}^{-1}$.

\subsubsection{Internal Standard}

HDI was used as internal standard (IS) for MDI determination and 1-Naphthyl was used as IS for TDIs determination. Solutions of HDI and 1-Naphthyl were prepared with a concentration of 2500 and $5000 \mu \mathrm{g} \mathrm{mL}-1$ in DCM, respectively. Intermediate standard solutions were prepared by dilution in AcN:DMSO and adding $100 \mu \mathrm{L}$ of $1,2-$ PP. These solutions were derivatized in the same conditions as the standards and samples. Working solutions were prepared by dilution with $\mathrm{AcN}$. The same concentration of the internal standard solution was added to all working solutions.

\subsubsection{Sample treatment}

Samples were prepared by adding adhesive or spraying foam into DCM. Intermediate solutions were prepared by dilution in AcN:DMSO and adding $100 \mu \mathrm{L}$ of 1,2-PP. These solutions were derivatized for two hours. Working solutions were prepared by dilution with AcN.

All standard solutions were filtered into a vial by a GHP 0.2 $\mu \mathrm{m}$ syringe filter.

\subsection{Chromatographic condition of UPLC-UV-Flu}

Acquity Ultra Performance LC system from Waters was used for the analysis of target compounds with an Acquity UPLC BEH C18 $(150 \times 2.1 \mathrm{~mm} ; 1.7 \mu \mathrm{m})$ column. The UPLC system has a Photo Diode Array detector (PDA) (Waters, Milford, MA, USA) acquiring at $240 \mathrm{~nm}$ and $254 \mathrm{~nm}$. Results are expressed in free-monomer isocyanate $(\% \mathrm{fm})$.
Table 1 summarizes the best chromatographic conditions achieved from pre-experiments.

\subsubsection{MAMA}

The mobile phase for MAMA derivatives was $80 \%$ acetonitrile and $20 \%$ water with $3 \%$ triethylamine adjusted to pH 3 with orthophosphoric acid. The column temperature was $30^{\circ} \mathrm{C}$ and flow rate at $0.3 \mathrm{~mL} \mathrm{~min}^{-1}$ during 15 minutes.

\subsubsection{1,2-PP}

For these solutions, the mobile phase consisted in two solvents. The first solution was $0.1 \%$ ammonium acetate adjusted to $\mathrm{pH} 6$ with acetic acid and the second solution was acetonitrile. Gradient elution using these two solutions was performed. The gradient was linear from $65: 35$ to $30: 70$ until 4.69 minutes, followed by another linear gradient to 5:95 until 5.16 minutes, isocratic elution with 5:95 during additional 1.87 minutes returning to initial condition with a total run time of 10 minutes. The column temperature was $40^{\circ} \mathrm{C}$ and the flow was $0.4 \mathrm{~mL} \mathrm{~min}^{-1}$.

\subsection{3. $D B A$}

The mobile phase for these solutions contained acetonitrile/water/formic acid (v/v/v) (A) 5/95/0.05 and (B) $95 / 5 / 0.05$. Elution was performed using a linear gradient from $40 \% \mathrm{~B}$ to $100 \% \mathrm{~B}$ in 12 minutes, followed by isocratic elution with $100 \% \mathrm{~B}$ for the additional 3 minutes. The column temperature was $30^{\circ} \mathrm{C}$ and flow was $0.35 \mathrm{~mL} \mathrm{~min}^{-1}$.

\subsection{Design of Experiments}

In the present work three variables were identified as potential parameters affecting the chromatographic conditions for isocyanate analysis. The three independent variables (controllable factors) were column temperature (Tcol), flow (flow) and percentage of ammonium acetate in solvent $A$ of the mobile phase (sol.). Each variable was studied at two levels. In order to evaluate and screen the complete set of main factors and their corresponding interactions a full factorial design with two levels for each factor was used. The levels of each factor, as well as the coded symbol and correspondent units, are summarized in Table 2. To control drift and to avoid biases, a full factorial design was carried out in a pseudo-random order, using "flow" as a blocking variable. A total of 8 experiments were performed in triplicate in a random sequence, in which each was injected two times. So, the experiences were reordered taking into account decreasing the total run time and, consequently, the amount of mobile phase. The design matrix is presented by standard order (Table 3), together with the results obtained for the two dependent variables analyzed (responses: peak area and resolution between two adjacent peaks). With the study of peak area, the objective was to maximize the response for detecting the higher content possible.

To reinforce the screening process, the study of the

Table 1. Overview of styles and font sizes used in this template.

\begin{tabular}{|c|c|c|c|c|c|}
\hline $\begin{array}{c}\text { Derivatization } \\
\text { Agent }\end{array}$ & Detector & Solvent A & Solvent B & $\begin{array}{c}\text { Flow } \\
\left(\mathrm{mL} \mathrm{min}^{-1}\right)\end{array}$ & Column Temperature \\
\hline MAMA & $\begin{array}{c}\text { PDA } \\
254 \mathrm{~nm}\end{array}$ & $\begin{array}{c}\text { 3\% Triethylamine } \\
\mathrm{pH}=3.0\end{array}$ & Acetonitrile & 0.33 & $30^{\circ} \mathrm{C}$ \\
\hline 1,2-PP & $\begin{array}{c}\text { PDA } \\
254 \mathrm{~nm}\end{array}$ & $\begin{array}{c}0.1 \% \mathrm{NH}_{4} \mathrm{Ac} . \\
\mathrm{pH}=6.0\end{array}$ & Acetonitrile & 0.4 & $40^{\circ} \mathrm{C}$ \\
\hline DBA & $\begin{array}{c}\text { PDA } \\
254 \mathrm{~nm}\end{array}$ & $\begin{array}{c}\mathrm{AcN} / \mathrm{H}_{2} \mathrm{O} / \mathrm{HCOOH} \\
5 / 95 / 0.05\end{array}$ & $\begin{array}{c}\mathrm{AcN} / \mathrm{H}_{2} \mathrm{O} / \mathrm{HCOOH} \\
95 / 5 / 0.05\end{array}$ & 0.35 & $30{ }^{\circ} \mathrm{C}$ \\
\hline
\end{tabular}


Table 2. Controllable factors (independent variables) with the coded symbol, units and the corresponding levels.

\begin{tabular}{lllll}
\hline & \multicolumn{1}{c}{ Factor } & & \\
\hline Variable & Coded symbol & Units & Low (-) \\
Column Temperature & $\mathrm{T}_{\text {col }}$ & ${ }^{\circ} \mathrm{C}$ & 30 & High (+) \\
Flow & Flow & $\mathrm{mL} \mathrm{min}^{-1}$ & 0.3 \\
Solvent & Sol. & $\%$ NH H$_{4}$ Ac. & 0.01 & 0.1 \\
\hline
\end{tabular}

Table 3. Factorial design matrix with corresponding results for peak area and resolution between peaks. Note: the letters $A$, $B$ and $C$ correspond respectively to column temperature (Tcol), flow (flow) and percentage of ammonium acetate in solvent A of the mobile phase (sol.). Low and high levels were denoted by “_" and " + ", respectively.

\begin{tabular}{|c|c|c|c|c|c|c|c|c|c|c|c|c|}
\hline \multirow[b]{3}{*}{$(1)$} & \multicolumn{3}{|c|}{ Factors } & \multicolumn{9}{|c|}{ Peak area per isocyanate } \\
\hline & \multirow{2}{*}{$\frac{A}{-}$} & \multirow{2}{*}{$\frac{B}{-}$} & C & \multicolumn{3}{|c|}{ 2,6-TDI } & \multicolumn{3}{|c|}{ 2,4-TDI } & \multicolumn{3}{|c|}{ MDI } \\
\hline & & & - & 955363 & 956056 & 961789 & 4513670 & 4522689 & 4502456 & 1158609 & 1151364 & 1150963 \\
\hline $\mathrm{a}$ & + & - & - & 964051 & 965921 & 943387 & 4495047 & 4509529 & 4486964 & 1060813 & 1055479 & 1076169 \\
\hline$b$ & - & + & - & 721916 & 717805 & 721036 & 3373708 & 3384527 & 3384071 & 861751 & 866549 & 818431 \\
\hline$a b$ & + & + & - & 722704 & 721636 & 723888 & 3377115 & 3384068 & 3383109 & 828270 & 831404 & 829637 \\
\hline c & - & - & + & 1006489 & 1004554 & 1004636 & 4613640 & 4603758 & 4612637 & 1166362 & 1163986 & 1168146 \\
\hline $\mathrm{ac}$ & + & - & + & 1004989 & 1004476 & 1004450 & 4601103 & 4592920 & 4590992 & 1076628 & 1089310 & 1090145 \\
\hline $\mathrm{bc}$ & - & + & + & 744180 & 738828 & 751788 & 3453850 & 3454082 & 3458858 & 872285 & 870533 & 874780 \\
\hline \multirow[t]{2}{*}{$a b c$} & + & + & + & 750909 & 750530 & 748874 & 3446738 & 3442698 & 3444175 & 837155 & 830967 & 836409 \\
\hline & A & $\mathrm{B}$ & $\mathrm{C}$ & \multicolumn{4}{|c|}{ Resolution between 2,6-TDI and 2,4-TDI } & \multicolumn{5}{|c|}{ Resolution between 2,4-TDI and MDI } \\
\hline$(1)$ & - & - & - & 3.3915 & & 3.4091 & 3.5397 & & 7.0381 & 7.0463 & & 7.1022 \\
\hline a & + & - & - & 1.9474 & & 1.9382 & 2.1732 & & 9.2782 & 9.1930 & & 9.4726 \\
\hline $\mathrm{b}$ & - & + & - & 1.8532 & & 1.8511 & 1.8973 & & 7.0609 & 6.8366 & & 6.9113 \\
\hline$a b$ & + & + & - & 2.0281 & & 2.0343 & 2.0734 & & 8.9455 & 8.6981 & & 8.8900 \\
\hline c & - & - & + & 2.2989 & & 2.2892 & 2.3131 & & 7.4277 & 7.3511 & & 7.1057 \\
\hline $\mathrm{ac}$ & + & - & + & 2.2180 & & 2.0364 & 2.0433 & & 9.8617 & 9.7837 & & 9.7918 \\
\hline $\mathrm{bc}$ & - & + & + & 2.0923 & & 2.1383 & 1.8169 & & 6.7289 & 6.6081 & & 6.6600 \\
\hline$a b c$ & + & + & + & 2.2171 & & 2.1026 & 2.1767 & & 9.4926 & 9.7007 & & 9.3122 \\
\hline
\end{tabular}

resolution between peaks will allow us to identify the best combination of factor levels that conduct to a better peak separation between the three isocyanates in the analysis. The resolution between peaks is defined by:

$$
R_{S}=\frac{2\left(t_{r 2}-t_{r 1}\right)}{w_{1}+w_{2}}
$$

where $t_{\mathrm{r} 1}$ and $t_{\mathrm{r} 2}$ are the retention time $\left(t_{\mathrm{r}}\right)$ for compounds 1 and 2 , in minutes, and $w_{1}$ and $w_{2}$ correspond to the peak widths (w) at half peak height for compound 1 and 2, respectively.

All the statistical analysis was performed using 'Minitab 16' (Minitab Inc., Cité Paradis, Paris, France) statistical software package, which included the analysis of variance (ANOVA) and the determination of the polynomial coefficients to be included in the predicting equations for both dependent variables. All ANOVA assumptions (model errors independent, normally distributed and constant variance) were validated through a complete residual analysis. Statistical significance was established at a $\mathrm{p}$-value $<0.05$ for all statistical tests applied.

\section{RESULTS AND DISCUSSION}

\subsection{Preliminary Experiments}

In this study, only diisocyanates were used so the amount of derivatization reagent had to be at least 2.1 times the amount of isocyanate. A kinetics reaction (results not shown) study was also performed to know the optimum time for the reaction to be complete.

To understand which derivatization reagent was most appropriate to determine isocyanates, preliminary experiences were performed. The layout of the chromatogram was used to assess the most suitable derivatizing agent and chromatographic conditions. The finest result for each derivatization reagent was summarized in Table 1.

Preliminary experiences revealed that 1,2-PP was the most appropriate derivatizing agent for isocyanate analysis. For this derivatization agent, the chromatogram layout showed less noise and better peak shape. The screening study was carried out only with 1,2-PP. 


\subsection{Design of Experiments}

A full factorial design (FFD) was performed to screen the chromatographic conditions and to provide a better understanding of the interactions between critical factors. The FFD analysis was run with the three factors shown in Table 2, namely column temperature $\left(\mathrm{T}_{\mathrm{col}}\right)$, flow (flow) and ammonium acetate percentage in solvent $\mathrm{A}$ of the mobile phase (sol.).

Results from preliminary experiences were used to select higher level (+). Lower level (-) was defined according to UPLC characteristics taking into account that UPLC columns are packed with solid phase particles smaller than those used in HPLC, which yields a lower theoretical plate height and higher analyte separation.

Two wavelengths for peak detection were selected (OSHA $254 \mathrm{~nm}$; EPA ctm-036A $240 \mathrm{~nm}$ ) [20], [21]. The quality of each result was defined by comparing peak areas or resolution between peaks under the same chromatographic conditions with detection at $254 \mathrm{~nm}$ and $240 \mathrm{~nm}$.

The analysis of variance (ANOVA) was chosen and applied directly to the responses (y) shown in Table 3, as well as to the transformed response given by a logarithmic transformation of variance $\left(-10 \log s^{2}\right)$, applied only to peak area. The first analysis allowed identifying which factors can significantly affect the two quality characteristics under study (peak area and resolution between adjacent peaks), obtained at appropriate wavelength (location effects), whereas the second analysis intends to investigate which factors affect the peak area variability (dispersion effects) [16], [23].

Tables 4 and 5 present the ANOVA results for the three isocyanates location effects, respectively for the responses peak area and the resolution between peaks. The significance of the factors and interactions, as well as the lack of fit, were evaluated through the $\mathrm{p}$-value and the F-distribution values. The analysis of the lack of fit (smallest p-value of 0.164 for 2,4-TDI peak area) allowed to conclude that the controllable factors chosen for this screening process were correct, allowing to explain the observed data variability expressed in Table 3. An exception was identified in Table 5 to the response "resolution between 2,6-TDI and 2,4-TDI peaks", where the lack of this quality parameter (lack of fit) is because all factors and interactions revealed high levels of significance ( $p$-value $<0.000$ ).

As can be seen in Table 4, and for all isocyanates, the flow was the most contributed factor that allows maximizing the peak area, with a percentage contribution to the total variability of $97.42 \%, 99.42 \%$ and $93.56 \%$, respectively for 2,6-TDI, 2,4TDI and MDI. The strong influence of this factor can be explained by the interaction analyte - mobile phase - stationary phase. Also, solvent distribution evidenced a significant effect over peak area, but with significantly less impact. Regarding column temperature, this factor does not reveal a significant effect on 2,6-TDI.

To select the chromatographic conditions, when two or more isocyanates are present, an evaluation of the resolution between peaks was performed (Table 5). With significant

Table 4. ANOVA results obtained for peak areas of the three isocyanates (2,6-TDI, 2,4-TDI and MDI). (SS - sum of squares; DoF - degrees of freedom; MS mean squares; $F_{o}$-statistical value given by factor and error mean squares ratio; $p$-value - level of significance of each factor)

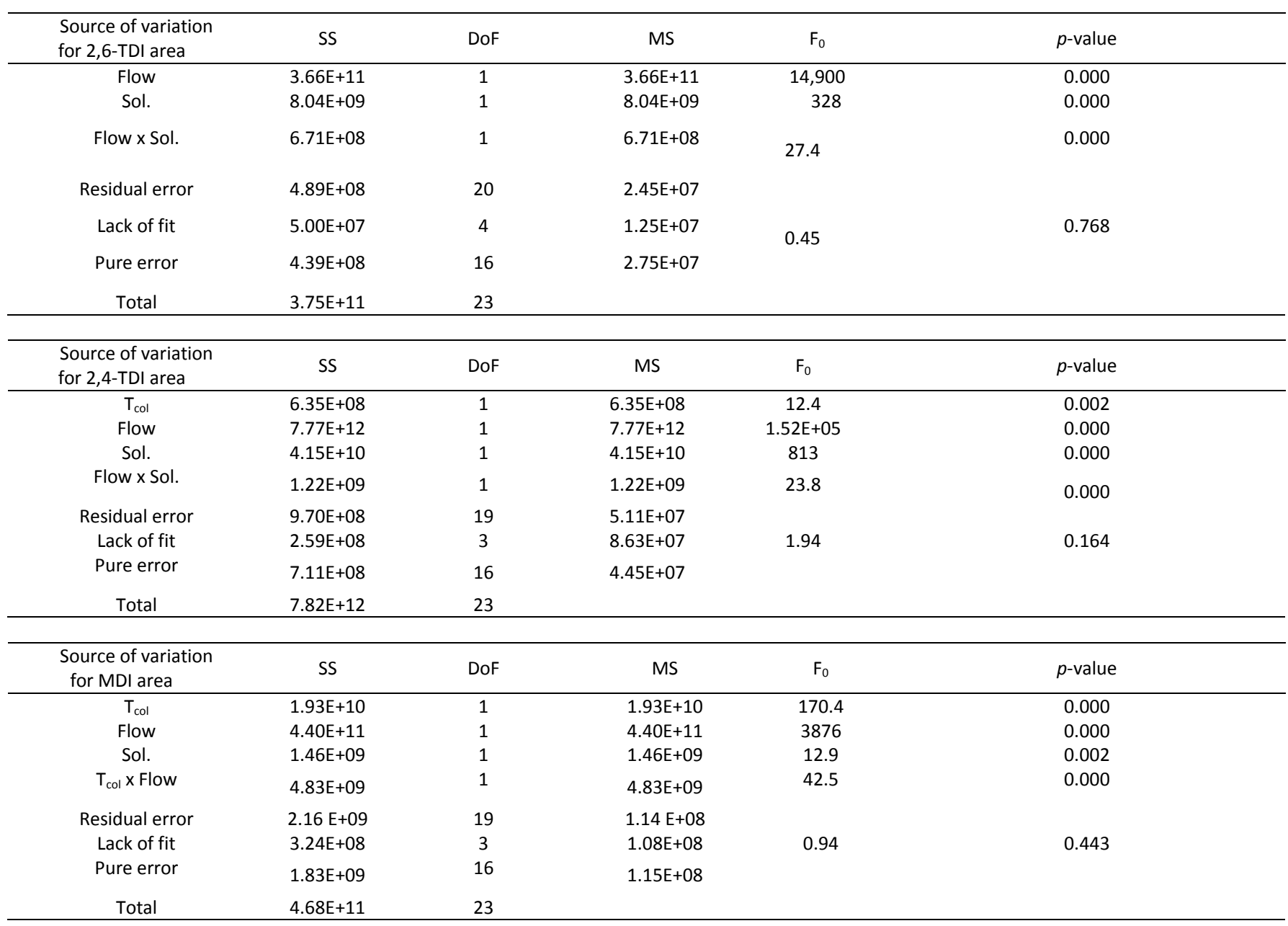


Table 5. ANOVA results obtained for resolution of 2,6-TDI - 2,4-TDI and 2,4-TDI - MDI. (SS - sum of squares; DoF - degrees of freedom; MS - mean squares; $F_{0}$-statistical values given by factor and error mean squares ratio; $p$-value - level of significance of each factor)

\begin{tabular}{|c|c|c|c|c|c|}
\hline $\begin{array}{l}\text { Source of variation for } \\
\operatorname{Res}_{(2,6-\mathrm{TDI}-2,4-\mathrm{TDI})}\end{array}$ & SS & DoF & MS & $\mathrm{F}_{0}$ & $p$-value \\
\hline $\mathrm{T}_{\text {col }}$ & 0.6344 & 1 & 0.6344 & 72.6 & 0.000 \\
\hline Flow & 1.1778 & 1 & 1.1778 & 134.9 & 0.000 \\
\hline Sol. & 0.2387 & 1 & 0.2387 & 27.3 & 0.000 \\
\hline $\mathrm{T}_{\text {col }} \times$ Flow & 1.4348 & 1 & 1.4348 & 164.3 & 0.000 \\
\hline $\mathrm{T}_{\text {col }} \times$ Sol. & 0.5378 & 1 & 0.5378 & 61.6 & 0.000 \\
\hline Flow x Sol. & 0.6689 & 1 & 0.6689 & 76.6 & 0.000 \\
\hline $\mathrm{T}_{\text {col }} \times$ Flow $\times$ Sol. & 0.5901 & 1 & 0.5901 & 67.6 & 0.000 \\
\hline Residual error & 0.1397 & 16 & 0.0087 & & \\
\hline Total & 5.4222 & 23 & & & \\
\hline $\begin{array}{l}\text { Source of variation } \\
\text { for } \operatorname{Res}(2,4-T D I-M D I)\end{array}$ & SS & DoF & MS & $\mathrm{F}_{0}$ & $p$-value \\
\hline $\mathrm{T}_{\mathrm{col}}$ & 33.9464 & 1 & 33.9464 & 2099.3 & 0.000 \\
\hline Flow & 0.8844 & 1 & 0.8844 & 54.7 & 0.000 \\
\hline Sol. & 0.4680 & 1 & 0.4680 & 28.9 & 0.000 \\
\hline $\mathrm{T}_{\text {col }} \times$ Sol. & 0.5338 & 1 & 0.5338 & 33.0 & 0.000 \\
\hline $\mathrm{T}_{\text {col }} \times$ Flow $\times$ Sol. & 0.1647 & 1 & 0.1647 & 10.2 & 0.005 \\
\hline Residual error & 0.2911 & 18 & 0.0162 & & \\
\hline Lack of fit & 0.0446 & 2 & 0.0223 & 1.4 & 0.265 \\
\hline Pure error & 0.2465 & 16 & 0.0154 & & \\
\hline Total & 36.2884 & 23 & & & \\
\hline
\end{tabular}

contributions, the flow (19.14\%), as well as the interaction between flow and column temperature $(23.88 \%)$ have a significant impact over the separation of both TDI isomers. For maximizing the resolution between 2,4-TDI and MDI the most significant factor obtained was column temperature, with a relative contribution of $92.74 \%$. These two peaks were well defined since they have a resolution value higher than 1.5. Thus, column temperature having such significant value, suggests that no other factor is important for this separation.

Based on the ANOVA results (Tables 4 and 5) five polynomial models were constructed, where only factors and interaction with a p-value smaller than 0.05 were chosen to the predictions under varying conditions (Table 6). The first three prediction models in coded terms considered the peak area for each of the three isocyanates (2,6-TDI, 2,4-TDI and MDI). Regarding peak resolution, the two predictive models obtained, in coded terms, are express in the last two lines of Table 6 , and corresponds to the peak resolution between 2,6-TDI and 2,4TDI, denoted by $\operatorname{Res}(2,6-\mathrm{TDI}-2,4-\mathrm{TDI})$, and between 2,4TDI and MDI, denoted by Res(2,4-TDI - MDI).

The quality of the developed models was assessed by the $\mathrm{R}^{2}$, predicted $R^{2}$ and adjusted $R^{2}$ values, shown in Table 6 . Since the reported values for these parameters are considerably higher
(>97\%), it means that the models developed through this Experimental Design strategy were well conducted and performed, allowing to predict the optimum chromatographic conditions of the three isocyanates in the study, at low concentration levels.

Still, within the same screening strategy, an analysis of variance was done using a $\log$ transformation of the peak area variance to identify which factors and/or interactions could reduce significantly the variability present within data (dispersion effects). This analysis could allow us to reach more robustness to peak area determination. The results for 2,4-TDI and MDI are shown in Figure 2 through a Pareto chart, where the significant factors and interaction bars appear above the vertical line ( $p$-value $<0.05)$. Each bar represents the estimate standardized effect in absolute value. For 2,6-TDI no significant effect was identified, meaning that the data variability due to the dispersion within each experiment couldn't be explained by any of these factors or interaction between them.

Regarding 2,4-TDI and MDI, it is clear that all factors (isolated or interactions between them) can contribute significantly to reduce the variability within the data, thus increasing data robustness and improve data accuracy.

Table 6. Final model equations with summary statistics.

\begin{tabular}{|c|c|c|c|c|}
\hline Variable & Final model & $\mathrm{R}^{2}$ & Predicted $\mathrm{R}^{2}$ & Adjusted $\mathrm{R}^{2}$ \\
\hline Area $_{2,6-\mathrm{TDI}}$ & $8.58 \mathrm{E}+5-1.23 \mathrm{E}+5$ Flow + 1.83E+4 Sol. $-5.29 \mathrm{E}+3$ Flow $x$ Sol. & $99.87 \%$ & $99.81 \%$ & $99.85 \%$ \\
\hline Area $_{2,4-T D I}$ & $3.98 \mathrm{E}+6-5.14 \mathrm{E}+3 \mathrm{~T}_{\text {col }}-5.69 \mathrm{E}+5$ Flow +4.16 Sol. $-7.12 \mathrm{E}+3$ Flow $x$ Sol. & $99.88 \%$ & $99.74 \%$ & $99.98 \%$ \\
\hline Area $_{\mathrm{MDI}}$ & $9.82 \mathrm{E}+5-2.84 \mathrm{E}+3 \mathrm{~T}_{\text {col }}-1.35 \mathrm{E}+5$ Flow $+7.80 \mathrm{E}+3 \mathrm{Sol} .+1.42 \mathrm{E}+4 \mathrm{~T}_{\text {col }} \times$ Flow & $99.54 \%$ & $99.26 \%$ & $99.44 \%$ \\
\hline $\operatorname{Res}_{(2,6-\mathrm{TDI}-2,4-\mathrm{TDI})}$ & $\begin{array}{l}2.2450-0.1626 \mathrm{~T}_{\text {col }}-0.2215 \text { Flow }-0.0997 \text { Sol. }+0.2445 \mathrm{~T}_{\text {col }} \times \text { Flow }+0.1497 \\
\mathrm{~T}_{\text {col }} \times \text { Sol. }+0.1669 \text { Flow } \times \text { Sol. }-0.1568 \mathrm{~T}_{\text {col }} \times \text { Flow } \times \text { Sol. }\end{array}$ & $97.42 \%$ & $94.20 \%$ & $96.30 \%$ \\
\hline $\operatorname{Res}_{(2,4-\mathrm{TDI}-\mathrm{MDI})}$ & $\begin{array}{l}8.1790+1.1893 \mathrm{~T}_{\text {col }}-0.1920 \text { Flow }+0.1396 \text { Sol. }+0.1491 \mathrm{~T}_{\text {col }} \times \text { Sol. }+0.0828 \mathrm{~T}_{\text {col }} \\
\mathrm{x} \text { Flow } \mathrm{x} \text { Sol. }\end{array}$ & $99.20 \%$ & $98.57 \%$ & $98.98 \%$ \\
\hline
\end{tabular}


Figure 2. Pareto plot evidencing the significant main factors and interaction for 2,4-TDI (left) and MDI (right), considering the log transformation of the peak area variance. Bars represent the standardized effect estimate in absolute value.

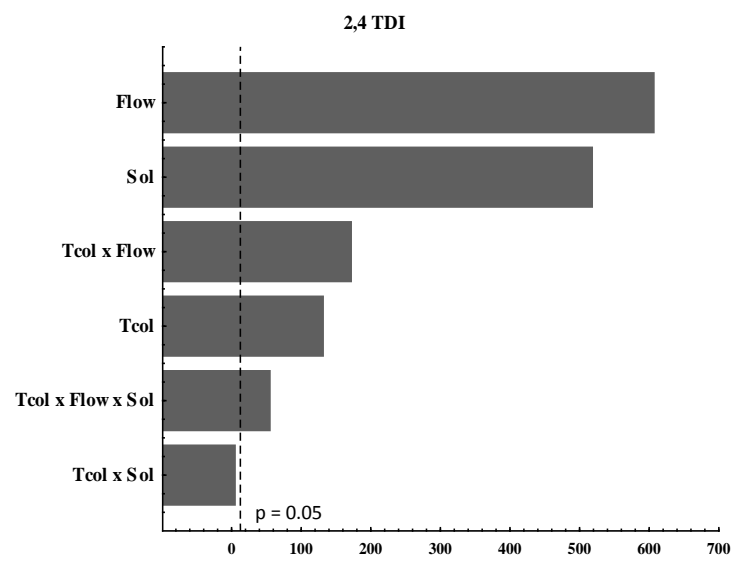

Note that for all analyses of variance involved in this study a complete residual analysis was performed for each one and models adequacy checked.

\subsection{Selection of the Optimal Conditions}

Based on the predictive models shown in Table 6, developed to improve free isocyanate determination at low concentrations, together with the Pareto charts information (Figure 2), a screening process was achieved to identify the best combination of factors level.

To reach a more accurate definition of the isocyanates peak, a maximization of the three dependent responses (peak area, resolution and variability) must be achieved. So, the signal of the isolated factors coefficient in the model equations (Table 6) indicates, as the best combination: column temperature at 30 ${ }^{\circ} \mathrm{C}$; flow at $0.3 \mathrm{~mL} \mathrm{~min}^{-1}$; and solvent at $0.1 \%$ NH4Ac.. The only exceptions identified were for both peaks resolutions, where for Res (2,6-TDI - 2,4-TDI) the solvent indicates the use of the low level $(0.01 \%$ NH4Ac.) and for Res (2,4-TDI - MDI) a column temperature of $40{ }^{\circ} \mathrm{C}$. The first conflict (sol.) could be ignored since the solvent shows the smaller $\mathrm{F}$ value within ANOVA Table $\left(\mathrm{F}_{0}=27.3\right)$, with a relative contribution to the total variability less than $2 \%$. Regarding the second conflict $\left(\mathrm{T}_{\text {col }}\right.$ at $\left.40{ }^{\circ} \mathrm{C}\right)$, the authors considered it important to analyse this situation in a different way. While the column temperature has a relative contribution smaller than $4 \%$ for the peak area of both isocyanates (2,4-TDI and MDI), the resolution between these two peaks shows the column temperature to be the most contributive factor with $92.7 \%$. The retention time when the column temperature was $40{ }^{\circ} \mathrm{C}$; a flow of $0.3 \mathrm{~mL} \mathrm{~min}^{-1}$; and a solvent of $0.1 \%$ NH4Ac. was $2.6 \mathrm{~min}$. for 2,4-TDI and 3.3 min. for MDI at $240 \mathrm{~nm}$. These were considered to be the optimum values for the 2,4-TDI and MDI resolution.

To analyse the significant effects of interactions and those considered on the $\log$ transformation of the variance, an analysis of means (ANOM) was performed but no level conflict

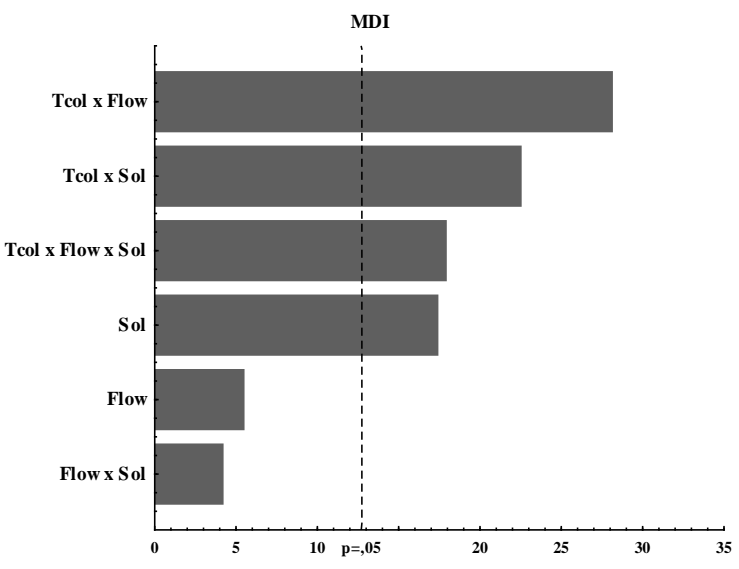

identified (results not shown).

\subsection{Method Performance}

Since validation guidelines were not established for isocyanates analysis determined by UPLC neither 1,2-PP use as a derivatizing agent, an In-house single laboratory validation procedure based on the Codex Alimentarius [24] was implemented to achieve the performance of the method. Parameters such as calibration curve, linearity, working range, limits of detection and quantification, reproducibility and recovery were evaluated and the method to analyze isocyanates present in materials in contact with foodstuff validated.

The linearity of the method was evaluated with calibration curves using 40 calibration points for MDI and 15 calibration points for TDI within the corresponding working range (Table 7).

The correlation coefficient was higher than 0.999 for both compounds. The limit of quantification was used to assess method sensitivity. The calculation of limit of detection (LOD) and limit of quantification (LOQ) values were based on the calibration curve [25]. All experimental values were within the acceptance criteria (residual standard deviation (RSD) $<15 \%$ ).

Due to lack of reference material, trueness was estimated, in terms of internal standard recovery, by evaluating the several concentration levels during ten days for MDI analysis and five days for TDI.

\subsection{Applicability}

The results of method applicability are presented in Table 8 . Samples, polyurethane adhesives based on TDI and MDI and polyurethane foams MDI based, obtained from the manufactures, produced in different days, were analyzed.

In TDI analysis the internal standard used was 1-naphthyl and all quantifications were determined with TDI/1-naphthyl ratio. The peaks were well resolved since the retention time was $2.42 \mathrm{~min}$ for 2,6-TDI, $2.66 \mathrm{~min}$ for 2,4-TDI and $3.07 \mathrm{~min}$ for 1 -

Table 7. Method performance.

\begin{tabular}{|c|c|c|c|c|c|c|c|c|}
\hline & Calibration curve & Linearity & Wo & $\begin{array}{l}\text { ing Range } \\
\mathrm{g} \mathrm{mL}^{-1} \text { ) }\end{array}$ & $\begin{array}{c}\text { LOD } \\
\left(\mu \mathrm{g} \mathrm{mL}^{-1}\right) \\
\end{array}$ & $\begin{array}{c}\text { LOQ } \\
\left(\mu \mathrm{g} \mathrm{mL}^{-1}\right)\end{array}$ & Reproducibility (\%) & $\begin{array}{c}\text { Recovery } \\
\text { (\%) }\end{array}$ \\
\hline MDI & $y=1 E+08 x+0.0113$ & 1 & 0.01 & 10.00 & 0.08 & 0.25 & 11 & 101.32 \\
\hline TDI & $y=3 E+08 x+0.0213$ & 0.9998 & 0.01 & 4.95 & 0.11 & 0.33 & 2 & 100.20 \\
\hline
\end{tabular}


Table 8. Quantification of free isocyanate content from polyurethane prepolymers in several matrices.

\begin{tabular}{|c|c|c|c|c|c|c|c|c|c|}
\hline & & Calibration curve & Linearity & $\begin{array}{l}\text { Working } \\
\text { Range } \\
\left(\mu \mathrm{gL}^{-1}\right)\end{array}$ & $\begin{array}{c}\text { LOD } \\
\left(\mu \mathrm{g} \mathrm{mL}^{-1}\right)\end{array}$ & $\begin{array}{c}\mathrm{LOQ} \\
\left(\mu \mathrm{g} \mathrm{mL}^{-1}\right)\end{array}$ & $\begin{array}{c}\text { Repeatability } \\
(\%)\end{array}$ & $\begin{array}{c}\text { Content } \\
(\%)\end{array}$ & $\begin{array}{c}\text { Recovery } \\
(\%)\end{array}$ \\
\hline \multirow{2}{*}{ TDI } & Adhesive 1 & $y=2 E+08 x+0.0085$ & 0.9995 & $0.01-7.87$ & 0.29 & 0.86 & 4.3 & $1.54 \pm 0.56$ & 100.65 \\
\hline & Adhesive 2 & $y=3 E+08 x+0.1301$ & 0.9992 & $0.01-9.89$ & 0.46 & 1.40 & 5.0 & $0.76 \pm 0.16$ & 100.57 \\
\hline \multirow{7}{*}{ MDI } & Adhesive 3 & $y=1 E+08 x-0.0133$ & 0.9997 & $0.01-9.96$ & 0.26 & 0.79 & 2.8 & $4.86 \pm 1.25$ & 106.57 \\
\hline & Adhesive 4 & $y=1 E+08 x-0.0133$ & 0.9997 & $0.01-9.96$ & 0.26 & 0.79 & 3.4 & $5.90 \pm 0.83$ & 105.00 \\
\hline & Adhesive 5 & $y=1 E+08 x-0.0133$ & 0.9997 & $0.01-9.96$ & 0.26 & 0.79 & 0.8 & $4.57 \pm 0.11$ & 111.72 \\
\hline & Adhesive 6 & $y=1 E+08 x-0.0133$ & 0.9997 & $0.01-9.96$ & 0.26 & 0.79 & 3.0 & $3.31 \pm 1.62$ & 105.49 \\
\hline & Foam 1 & $y=1 E+08 x-0.0205$ & 0.9995 & $0.01-9.01$ & 0.33 & 1.01 & 0.5 & $0.93 \pm 0.02$ & 100.40 \\
\hline & Foam 2 & $y=1 E+08 x-0.0205$ & 0.9995 & $0.01-9.01$ & 0.33 & 1.01 & 1.4 & $1.06 \pm 0.07$ & 101.38 \\
\hline & Foam 3 & $y=1 E+08 x-0.0205$ & 0.9995 & $0.01-9.01$ & 0.33 & 1.01 & 0.4 & $0.87 \pm 0.10$ & 100.62 \\
\hline
\end{tabular}

naphthyl as shown in Figure 3. The validation parameters were adequate with good linearity and recovery between our acceptance criteria (80 \%-120\%) can be observed.

HDI was the internal standard for MDI analysis. The retention time was, for HDI $2.45 \mathrm{~min}$ and $3.34 \mathrm{~min}$ for MDI so the peaks were well resolved (Figure 3). The recoveries were good for the MDI/HDI ratio so for these peaks there isn't a matrix or other interferences. The linearity was good for both matrixes MDI based.

A $2 \% \mathrm{fm}$-TDI maximum was found in TDI based adhesives. Fm-MDI content in adhesives ranged from $3 \%$ to $6 \%$. The amount of $\mathrm{fm}-\mathrm{MDI}$ in MDI based foams was below $1 \%$.
These values were compared with the expected values theoretically calculated and experimental values obtained by titration carried out in another laboratory. The values were not statistically different.

The methods proved to be efficiency because real samples content have the expected value [26].

Another advantage is the fact that these pre-polymers can be used in agglomerated cork stoppers industries.

\section{CONCLUSION}

Three derivatization agents (MAMA, DBA and 1,2-PP) were studied before the experimental design. 1,2-PP was chosen for
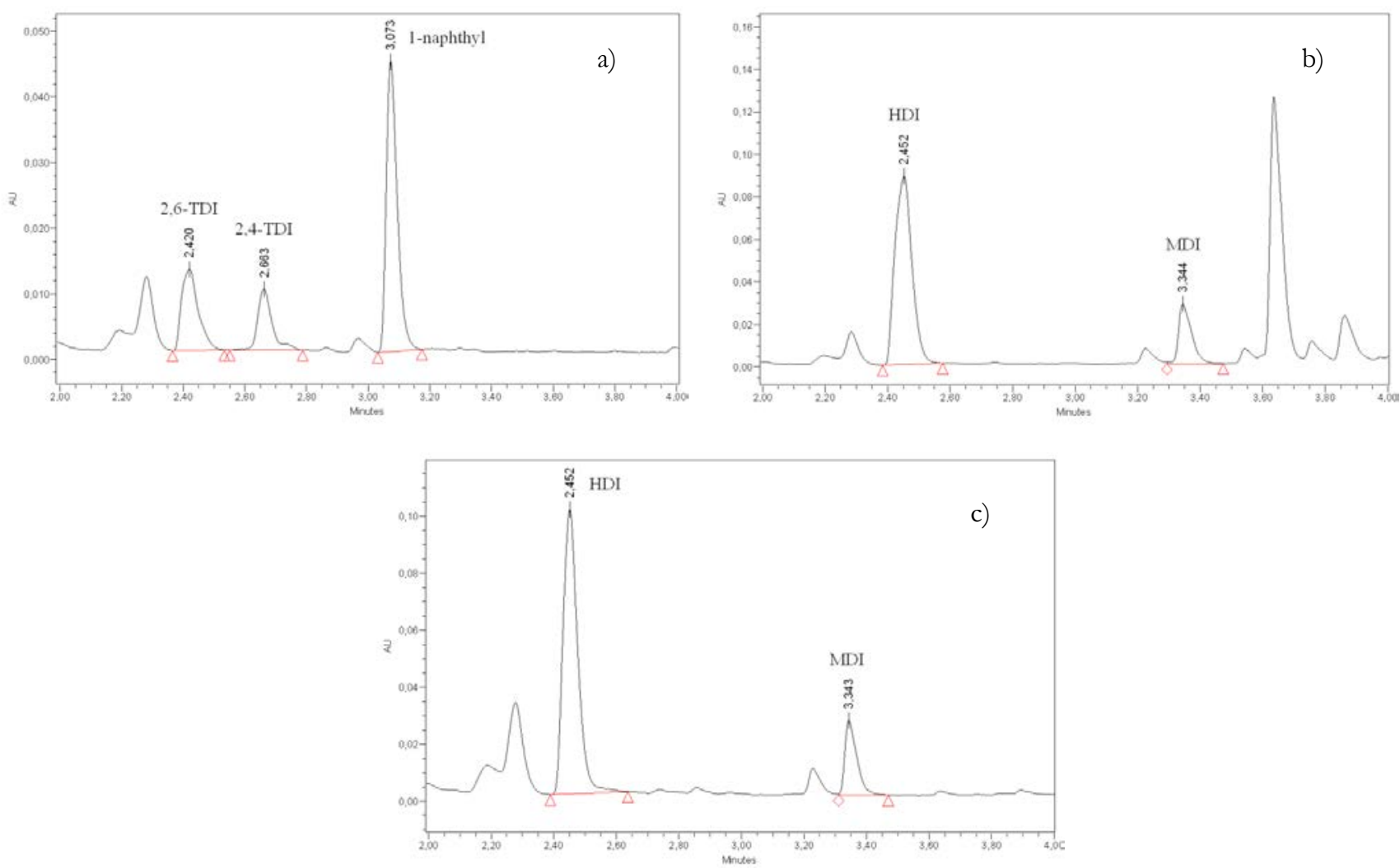

Figure 3. Chromatogram of three real samples. a) Chromatogram of adhesive based on TDls; b) Chromatogram of foam based on MDI; c) Chromatogram of adhesive based on MDI. 
DOE application since it was the one that provided the best results in preliminary experiments.

The chromatographic method to determine free diisocyanates, at low concentration levels, should be performed with a column temperature of $30{ }^{\circ} \mathrm{C}$, a flow of $0.3 \mathrm{~mL} \mathrm{~min}{ }^{-1}$ and a concentration of ammonium acetate in the mobile phase of $0.1 \% \mathrm{NH} 4 \mathrm{Ac}$. The best wave length of the PDA detector to quantify MDI was $254 \mathrm{~nm}$ and for the TDIs it was $240 \mathrm{~nm}$.

Two methods were developed since the MDI internal standard (HDI) has the same retention time of 2,6-TDI. Also, from the design of experiments, the best combination for peak maximization was different for the analysis of MDI and TDIs. The results demonstrated that methods are efficient to determine isocyanates at low concentration levels in the material in contact with foodstuff. For both methods, linearity was higher than 0.999 and recovery approximately $100 \%$.

The studied pre-polymers are adequate as "food grade" materials, such as agglomerated cork stoppers among others, since the fm-isocyanate content is under the theoretical levels.

\section{ACKNOWLEDGMENTS}

Catarina André and Inês Delgado acknowledge the financial support from the Portuguese Innovation Agency (ADI) in the frame of project QREN 5012-LIRACork. This work was supported by the Portuguese Innovation Agency (ADI) in the frame of project QREN 5012-LIRACork.

\section{REFERENCES}

[1] C.E. Liège, International Code of Cork Stopper Manufacturing Practices, 1996.

[2] A.P. Damant, S.M. Jickells, L. Castle, Liquid chromatographic determination of residual isocyanate monomers in plastics intended for food contact use, J. AOAC Int. 78, (1995), pp. 711-719.

[3] Health and Safety Laboratory, Methods for the determination of hazardous substances \#25/3 Organic isocyanates in air (MDHS 25/3), (1999).

[4] British Standard, BS EN 13130-8:2004 - Materials and articles in contact with foodstuffs - Plastics substances subject to limitation - Part 8: Determination of isocyanates in plastics, (2004).

[5] U. S. Environmental Protection Agency (EPA), Method 207 Method for measuring isocyanates in stationary source emissions, (2006).

[6] P. Tremblay, J. Lesage, C. Ostiguy, H. Van Tra, Investigation of the competitive rate of derivatization of several secondary amines with phenylisocyanate (PHI), hexamethylene-1,6-diisocyanate (HDI), 4,4'-methylenebis(phenyl isocyanate) (MDI) and toluene diisocyanate (TDI) in liquid medium, Analyst. 128, (2003), pp. 142-149.

[7] K. Mastovska, Recent Developments in Chromatographic Techniques, in: Y. Picó (Ed.), Compr. Anal. Chem. - Vol. 51
Food Contam. Ans Residue Anal., 1st ed., Elsevier B.V., (2008), pp. 175-200.

[8] M.E. Swartz, Ultra Performance Liquid Chromatography (UPLC): An Introduction, Sep. Sci. Redefined. May, (2005), pp. 8-14.

[9] J.F. McGaughey, S.C. Foster, R.G. Merrill, Laboratory development and field evaluation of a generic method for sampling and analysis of isocyanates, EPA Report, 1995.

[10] T. Lundstedt, E. Seifert, L. Abramo, B. Thelin, A. Nyström, J. Pettersen, et al., Experimental design and optimization, Chemom. Intell. Lab. Syst. 42, (1998), pp. 3-40.

[11] B.J. Stojanović, Factorial-Based designs in liquid chromatography, Chromatographia. 76, (2013), pp. 227-240.

[12] D.B. Hibbert, Experimental design in chromatography: A tutorial review, J. Chromatogr. B. 910, (2012), pp. 2-13.

[13] W. Dewé, R.D. Marini, P. Chiap, P. Hubert, J. Crommen, B. Boulanger, Development of response models for optimising HPLC methods, Chemom. Intell. Lab. Syst. 74, (2004), pp. 263-268.

[14] X. Zhang, R. Wang, X. Yang, J. Yu, Central composite experimental design applied to the catalytic aromatization of isophorone to 3,5-xylenol, Chemom. Intell. Lab. Syst. 89, (2007), pp. $45-50$.

[15] A.C. Atkinson, R.D. Tobias, Optimal experimental design in chromatography, J. Chromatogr. A. 1177, (2008), pp. 1-11.

[16] D.C. Montgomery, Design and Analysis of Experiments, 5th ed, Jonh Wiley \& Sons, INC., 1997, ISBN: 0-471-31649-0.

[17] N. Akvan, H. Parastar, Second-order calibration for simultaneous determination of pharmaceuticals in water samples by solidphase extraction and fast high-performance liquid chromatography with diode array detector, Chemom. Intell. Lab. Syst. 137, (2014), pp. 146-154.

[18] C. André, F. Jorge, I. Castanheira, A. Matos, Optimizing UPLC isocyanate determination through a Taguchi experimental design approach, J. Chemom. 27, (2013), pp. 91-98.

[19] C.J. Purnell, R.F. Walker, Methods for the determination of atmospheric organic isocyanates. A review, Analyst, 110 (1985), pp. 893-905.

[20] OSHA Analytical Laboratory, Method No. 47: Methylene Bisphenyl Isocyanate (MDI), Salt Lake City, 1989.

[21] OSHA Analytical Laboratory, Method No. 42: Diisocyanates, Salt Lake City, 1989.

[22] U. S. Environmental Protection Agency (EPA), Ctm 036a Analysis of Isocyanates Liquid Chromatography - Diode Array / MSD, 2004.

[23] A. Ávila, E.I. Sánchez, M.I. Gutiérrez, Optimal experimental design applied to the dehydrochlorination of poly(vinyl chloride), Chemom. Intell. Lab. Syst. 77, (2005), pp. 247-250.

[24] WHO/FAO, Codex Alimentarius Commission Procedural Manual, 21st ed., Rome, Italy, 2013.

[25] P. Konieczka, J. Namiesnik, Quality Assurance and Quality Control in the Analytical Chemical Laboratory: A Practical Approach, CRC Press, 2009, ISBN 9781420082708.

[26] I. Delgado, C. André, A. Ramos, A.S. Matos, K. Stockham, S. Kumaran, et al., "Bracketing versus multipoint calibration in determination of isocyanates in agglomerated cork stoppers", Proc of XX IMEKO World Congress Green Growth, Sept. 9-14, 2012, Busan, Republic of Korea, vol 1, pp. 696-699. 